Aquaculture

September 2018, Volume 494, Pages 10-18

http://dx.doi.org/10.1016/j.aquaculture.2018.05.009

http://archimer.ifremer.fr/doc/00439/55090/

(c) 2018 Elsevier B.V. All rights reserved.

\title{
Evaluation of different tags on survival, growth and stress response in the flatfish Senegalese sole
}

\author{
Carballo Carlos ${ }^{1}$, Berbel Concha ${ }^{1}$, Guerrero-Cózar Israel ${ }^{1}$, Jiménez-Fernández Eduardo ${ }^{2}$, \\ Cousin Xavier ${ }^{3,4,5}$, Bégout Marie-Laure ${ }^{3}$, Manchado Manuel ${ }^{1{ }^{*}}$
}

${ }^{1}$ IFAPA Centro El Toruño, Junta de Andalucía, Camino Tiro Pichón s/n, 11500 El Puerto de Santa María, Cádiz, Spain

${ }^{2}$ CUPIMAR, Ctra. Carraca, s/n, Salina San Juan Bautista, San Fernando, Cádiz, Spain

${ }^{3}$ Laboratoire Ressources Halieutiques, Ifremer, Place Gaby Coll, 17137 L'Houmeau, France

${ }^{4}$ Laboratoire Adaptation et Adaptabilités des Animaux et des Systèmes, UMR MARBEC, Ifremer, Route de Maguelone, 34250 Palavas, France

${ }^{5}$ GABI, INRA, AgroParisTech, Université Paris-Saclay, 78350 Jouy-en-Josas, France

* Corresponding author : Manuel Manchado, email address : $\underline{\text { manuel.manchado@juntadeandalucia.es }}$

\begin{abstract}
:
Internal electronic tagging is a major issue in flatfish species due to the small size of abdominal cavity. In this study, three tag types, referred to as Nonatec, nano and mini, were evaluated in three weight classes of Senegalese sole: small $(0.3 \mathrm{~g})$, middle $(0.8 \mathrm{~g})$ and large $(2.0 \mathrm{~g})$. Tags were injected from the blind side and fish were carefully handled to minimize sharp movements. Tag losses were $8 \%$ in the small size class, between 5.2 and $15.1 \%$ in the middle size class and $2-4 \%$ in the large size class. The mortality rates ranged between 2.0 and $15.0 \%$ with the lowest values in the large size class. No negative effects of tags on growth (tagged vs non-tagged fish using a middle size class) were found after 57 days of culture. Four additional trials using mini tags in a large size class at industrial scale validated our experimental results. With respect to morphology, no differences in the area, ellipticity and circularity were found except for a slight higher aspect ratio index in mini- and nano-tagged soles when compared with untagged fish. A longitudinal analysis of growth using the tag type, sex and tag position (anterior, medium or posterior) as fixed factors revealed a significant and strong effect of sex, with females appearing significantly heavier (13.6\%) than males. In addition, the significant interactions between tag position and tag type with the time indicated a delayed growth of Nonatec-tagged fish and specimens with tags in the posterior section of abdomen. Expression analysis of stress-related genes revealed an activation of HPI axis and cellular stress defenses at 2 days just after tagging (dat) not evident at 11 dat. All these data indicate that soles can be successfully tagged at very small sizes both at experimental and industrial scales if tag type is properly selected and fish correctly handled. Moreover, sex and tag position are significant factors affecting growth that need to be controlled in longitudinal studies and selective breeding programs.
\end{abstract}




\section{Highlights}

- A methodology for internal tagging of small soles was developed and validated at experimental and industrial scales. Optimal tag retention and mortality rates were obtained and no negative effects on growth or morphology were detected. Longitudinal analysis using internal tags revealed a major effect of sex, tag type and tag position on growth. Expression analysis indicated no signs of chronic stress linked to internal tags.

Keywords : Flatfish, PIT-tags, Loss rates, Sex effect, Growth 


\section{Introduction}

Internal tags have become a valuable tool for longitudinal studies in fish aquaculture and ecology (Bégout et al., 2016; Cousin et al., 2012; Ferrari et al., 2014; Jepsen et al., 2005; Mahapatra et al., 2001). The PIT (Passive Integrated Transponder) tags are the most common devices since they can be used in large populations in an operational way. In the last years, PIT tags ranging between 6 and $12 \mathrm{~mm}$ in length and with a low weight ( 2-4\% tag/body weight ratio) have been successfully used in fish (Baras et al., 2000; Cousin et al., 2012; Jepsen et al., 2005; Ombredane et al., 1998). However, the great diversity in fish morphology requires that both the tag type and the injection procedure have to be specifically validated in a species- and class sizes-specific way and the effects on growth, survival, behavior and stress concurrently assessed. In pelagic fish, several studies reported optimal retention ratios $(>90 \%)$ with little or no effects on fish survival and growth although retention ratios were highly influenced by fish size (Acolas et al., 2007; Larsen et al., 2013; Mahapatra et al., 2001; Navarro et al., 2006; Soula et al., 2011). In flatfish, internal tagging is a bit more complicated by some methodological constraints due to the small size of the abdominal cavity and fish handling. Although specific procedures in large-size flatfish species such as plaice and turbot were optimized by accessing from the eyed-side (Moser et al., 2005; Oesau et al., 2013; Sparrevohn et al., 2014), specific methods need to be optimized in smaller and more ellipsoid flatfishes such as soles.

The Senegalese sole (Solea senegalensis) is an economically important flatfish species in Southern Europe. The aquaculture production under recirculation aquaculture systems (RAS) grew exponentially in the last five years. However, some bottlenecks related with reproduction success and size dispersion still persist (Morais et al., 2016) that require advanced studies to identify hatchery and management solutions. Up to now, the visible implant elastomer (VIE) has 
been the most used individual tagging method in this species to evaluate growth performance and to study social interactions in population (Salas-Leiton et al., 2010a; Salas-Leiton et al., 2010b). However, the use of distinct patterns based on color and shape applied on the blind side of juveniles was prone to errors due to the complexity to read and interpret the VIE tagging patterns. Hence, new methodologies based on internal electronic tags are required to provide unique identification codes, easy to read, functional for long periods and that can be applied to a large range of fish sizes. Currently, PIT tags are intramuscularly applied to sole breeding stocks (Anguís and Cañavate, 2005) and juveniles in behavioral trials (Ibarra-Zatarain et al., 2016). However, an intraperitoneal tagging method needs to be optimized and validated in small size soles just at the beginning of pre-ongrowing stage $(2-5 \mathrm{~g})$ in order to design and implement selective breeding programs. Moreover, the analysis of some expression markers related to the hypothalamus-pituitary-interrenal axis and cellular stress (Benitez-Dorta et al., 2017; BenitezDorta et al., 2013; Manchado et al., 2008; Montero et al., 2015; Salas-Leiton et al., 2012). will allow to assess the impact on animal welfare and the suitability to evaluate complex traits in longitudinal studies.

The aim of this study was to evaluate the suitability of three internal PIT tags of different sizes and to monitor effects on tag losses, mortality, growth, morphology and expression of some stress-related genes in sole. This evaluation included different fish weight classes and were performed both at experimental and industrial scales over a long term. The data gathered highlight the importance of individual tagging to evaluate growth performance in young sole juveniles controlling some other variables such as sex and tank effects which both have a profound impact on the statistical models and results accuracy. 


\section{Material and Methods}

\subsection{Tagging protocol and fish trials}

All soles used in this study belonged to the same spawning batch and they were supplied by CUPIMAR (San Fernando, Cadiz, Spain). All procedures were authorized by Bioethics and Animal Welfare Committee of the IFAPA and registered with number 06-11-15-337 by National authorities for regulation of animal care and experimentation.

Before carrying out the fish trials, the tag injection procedure was optimized and adapted to the morphology and behaviour of sole. All staff was trained to acquire skills in needle, tags and fish handling prior to the trial. Specimens were fasted for 1 day before tagging and they were deeply anesthetized before handling (phenoxyethanol, $150 \mathrm{ppm}$ ). Before injection, PIT tags were disinfected by immersion in $70 \%$ ethanol followed by a distilled water washing. Animals were flipped blind side up and a small incision in the posterior part of the abdominal cavity was done using a G18 or G19 needle (previously disinfected in $70 \%$ ethanol and rinsed in distilled water). The size of the incision was wide enough to allow for the tag injection by exerting a bit pressure with forceps (Suppl. file 1). Large incisions were avoided to accelerate the healing of the wounds. When a high number of specimens were tagged, the needles were periodically changed to be sharp enough to easily cut through the skin and muscle wall. The introduction of the tag was always parallel to the ventral fin by gently pressing on the top of the abdominal cavity to control tag injection and avoid any damage to internal organs. After completing this procedure, the tag was softly displaced in the abdominal cavity to move away from the incision. Once completed, the incisions were covered with iodine gel (betadine gel) to prevent infections and to facilitate wound healing. Thereafter fish were transferred back to their home tanks in their natural position (standing on the blind side) to avoid agitation after anaesthesia recovery. To 
minimize movements in the tank after tagging, animals were fasted for $24 \mathrm{~h}$. Average handling time required to tag one fish ranged between 30 and 60 s.

To evaluate the effects of PIT tags on survival, tag loss rate, growth and stress responses, different tagging sessions were carried out that resulted in three experimental trials to evaluate: i) tag losses and survival (experiment 1), ii) growth performance (experiment 2a) and iii) effects on gene expression (experiment 2b). A last experiment was conducted at an industrial scale (experiment 3) In all cases, the above procedure was followed paying special attention to the following points: a) animals were deeply anesthetized before handling and during all the procedure; b) careful return to the home tank in natural position to avoid agitation; c) further fish handing was reduced as much as possible by using self-cleaning tanks $(1.0 \mathrm{~m} \times 0.5 \mathrm{~m}$, Volume $=$ $0.05 \mathrm{~m}^{3}$ ) and just manipulating belt-feeders once day.

Experiment 1: In the first tagging session, a trial to evaluate tag losses and fish survival was carried out. Three PIT tag types were tested (Suppl. file 1): a) $\operatorname{Nonatec}^{\mathrm{TM}}$ (1x6 mm, $7.25 \mathrm{mg}$ ); b) Nano transponder (ID-100A/1.25, Trovan; 1.25x7 mm, 25mg); c) Mini transponder (ID100A/1.4 Trovan, $1.4 \times 8 \mathrm{~mm} 30 \mathrm{mg}$ ). Moreover, these three tags were injected in three weight classes: small (average $0.3 \mathrm{~g}$ ), middle (average $0.8 \mathrm{~g}$ ) and large class (average $2.0 \mathrm{~g}$ ). All individuals were randomly selected and assigned to each tag experimental group by class size. Mean weights for each tag type and size class are presented in Table 1. In the small weight class, only the Nonatec tags could be evaluated since the small size of the abdominal cavity impeded the injection of larger tags. After tagging, all individuals $(n=25)$ were placed in one tank. In the middle size class, soles were injected with the three tag types and dispatched in triplicate tanks (the total number of individuals by tag type ranged between 145 and 157, with 45 to 53 soles per tank, 9 tanks in total). In the large size class, 50 individuals for each tag type were injected and 
distributed into separate tanks (one tank per tag type, 3 tanks in total). For each fish, weight, length and surgeon identity (five persons in total) were recorded. After tagging, soles were dispatched in self-cleaning rectangular tanks in an open flow circuit with one water renewal each two hours that ensured a high water quality and cleanness of surfaces. Food ( $2 \%$ of fish biomass; Gemma Micro Skretting, Spain) was supplied from the second day after tagging onwards by using 12h-belt feeders. Tag losses and mortality were recorded daily for 15 days. Water temperature and salinity were $18.1 \pm 0.5{ }^{\circ} \mathrm{C}$ and $37 \mathrm{ppt}$, respectively. A treatment with hydrogen peroxide (100 ppm) was also weekly applied to facilitate wound healing and prevent diseases. Weight was automatically registered for Nano and Mini transponders using the FR-200 FishReader W (Zeuss, Trovan, Spain). For Nonatec tagged fish, the hand-held Bluetooth reader was used to read the tag and weight was manually inserted in a spreadsheet.

In a second tagging session, two trials were carried out.

Experiment 2a: Firstly, the effects of the three PIT tag types on growth performance and morphology were evaluated using a middle weight class $(1.0 \mathrm{~g})$. A set of 360 soles was tagged using the Nonatec, nano- and mini transponders and distributed separately into nine tanks ( $\mathrm{n}=40$ per tank). Animals were daily inspected for ten days and only 30 individuals with completely healed wounds were randomly selected and maintained in each tank. Then, thirty untagged soles from the same larval batch and of similar size were added to each tank (Table 2). Food (2\% fish biomass; Gemma Micro Skretting, Spain) was supplied from the second day after tagging onwards by using 12h-belt feeders. The tanks were daily inspected for tag losses and mortality. Weight and standard length (from the mouth until the base of caudal fin) were recorded at 20, 36 and 57 days after adding untagged fish. Body width (in the maximal width point excluding the dorsal and ventral fins) was measured at the end of the trial. In the first biometry (at 20 days, i.e. 
30 days post tagging), the presence of a scar on the blind side was used to distinguish untagged fish from those that had lost their tag. In the last two samplings, due to the absence of any skin lesion, the expected number of tagged fish was confirmed by tag reading. In the case of Nonatec reader, the weakness of this system to accurately detect the tag signal forced us to pass all fish at least three times through the reader until all tagged individuals were identified. Due to this intense handling of Nonatec-tagged soles, some animals $(n=9)$ developed lesions in the caudal fin area and were excluded from the growth analysis. Water temperature and salinity were 19.2 $\pm 0.5{ }^{\circ} \mathrm{C}$ and $35 \mathrm{ppt}$, respectively. As indicated above, a hydrogen peroxide (100 ppm) treatment was weekly applied. At the end of the experiment, all fish were euthanized using phenoxyethanol (300 ppm), and then measured, photographed using a panasonic camera (DMC F3 Lumix $12 \mathrm{Mpx}$ ) and under X-rays to examine tag position in the body cavity. Finally, animals were visually sexed by opening the abdominal cavity.

Experiment 2b: Moreover, a second trial was done in this second tagging session to assess the effects on gene expression. For this purpose, four tanks were established, each of one contained soles tagged with Nonatec $(n=6)$, nano $(n=6)$ and $\operatorname{mini}(n=6)$ transponders as well as untagged fish $(\mathrm{n}=6)$. Animals were sampled at 2 and 11 days after tagging (dat). They were euthanized as indicated above and rapidly dissected to collect liver and brain. Samples were fixed in RNA-later (Invitrogen) for gene expression analysis and stored at $-80^{\circ} \mathrm{C}$ until use.

Experiment 3: To validate the use of this tagging procedure in genetic breeding programs at industrial scale, four tagging sessions in collaboration with the company CUPIMAR were carried out. After staff training in the tagging procedure, four batches of soles ranging in weight between 1.7 and $6.6 \mathrm{~g}$ were established (Table 1). All animals were tagged using the mini transponder (Trovan) by just one surgeon. The tagging procedure of each sole batch spanned over 2-3 days 
and the number of tagged fish ranged between 428 and 786 individuals per batch. After tagging, animals of each batch were separately dispatched in self-cleaning circular tanks (surface $=0.8$ $\mathrm{m}^{2}$ ). Water temperature and salinity were $20.1 \pm 0.3{ }^{\circ} \mathrm{C}$ and $30 \mathrm{ppt}$, respectively. As previously described, a treatment with hydrogen peroxide (100 ppm) was weekly applied and the food provided from the second day after tagging onwards (2\% fish biomass). Tag losses and mortality were daily recorded for 15 days. Moreover, the batch of soles with average weight $1.7 \mathrm{~g}(\mathrm{n}=748$ as indicated in Table 1) was also monitored for growth performance. Fish were maintained separately for 15 days until wound healing and thereafter, all tagged fish were moved to a new tank and 6,000 untagged specimens of the same weight class were added. The tank was selfcleaning and rectangular $\left(6 \mathrm{~m}^{2}\right)$ and food was provided ad libitum. To evaluate the effects on growth and simplify handling for fish measurements with such a high number of fish in each tank, a subset of 50 tagged specimens were randomly selected to monitor fish growth. To select in a feasible way these tagged animals from the whole population, the list of tags was uploaded in a portable reader (LID 560, Trovan) and all fish were passed through it until identifying the complete subset of selected tagged fish. Then, fifty untagged animals were also randomly selected from the tank and all fish were weighted using FISH Reader (Zeus, Trovan). Biometries were carried out at 39,81, 113 and 148 days after the start of the experiment and all sole were put back to their home tank at the end of each biometry to maintain the fish population size constant.

\subsection{Data analysis}

Fish mortality was calculated as the percentage of dead fish relative to the total number of fish initially tagged in each tank. Tag loss rates were estimated as the number of free tags in the 
bottom of the tank relative to the total number of fish initially tagged. Before analysis, all data were checked for normality using the Shapiro-Wilk test and homogeneity of variance using Bartlett's test.

Statistical differences between tag losses using the middle size class were tested using a nonparametric Kruskal-Wallis test ( $\mathrm{n}=3$ tank replicates). The effect of surgeon on survival was tested using a chi-square test. The association between loss rates and weight was estimated by Spearman's rho parameter. All these data were analyzed using the software Prism 7 (Graphpad).

To determine the effects of tagging on growth, the specific growth rate (SGR) was individually computed in tagged fish in the three samplings (SGR20 from 0 to 20 days, SGR36 from 0 to 36 days and SGR57 from 0 to 57 days post-tagging) using the following formula SGR $\left(\% \mathrm{~d}^{-1}\right)=$ $\left(\ln \mathrm{W}_{\mathrm{f}}-\ln \mathrm{W}_{\mathrm{i}}\right) / \Delta \mathrm{t}^{*} 100 ;$ where $\mathrm{W}_{\mathrm{f}}, \mathrm{W}_{\mathrm{i}}$, and $\Delta \mathrm{t}$ represented final and initial weight and time interval, respectively. For untagged fish, the SGR was calculated using the weight geometric mean as defined in Marquez et al. (2015). To evaluate morphological traits, images at the end of the experiment were processed using ImageJ 2.0.0rc43/1.51e. To select the full sole area, the wand tool was used to fit as much as possible to the ellipsoid morphology of soles. In all cases, the selected area included a small fraction of dorsal, ventral and caudal fins (Suppl. file 1). Shape descriptors and ellipse fitting (Circularity [4pi*area/sqr(perimeter)], aspect Ratio [major axis/minor axis] and area were analyzed. For tag position, X-ray radiographies of the whole set of animals in each tank were read and the tag position was determined establishing three positions: anterior, if situated between the septum separating ocular and blind side and the gills; medium, if situated just behind the septum; and posterior, if situated just at the posterior part of the abdominal cavity (Suppl. File 2). 
To compare weight and length at the different sampling points between tagged and untagged fish, a General Linear Model (GLM) analysis was carried out including being tagged or not as fixed factor and tank as random factors using SPSS v23.0 (SPSS, Chicago, IL, USA). All data were tested for normality (Kolmogorov-Smirnov) and homogeneity of variances (Levene's test) and log-transformed when necessary. The GLM model was $Y_{\mathrm{ijk}}=\mu+\alpha_{\mathrm{i}}+\beta_{\mathrm{j}}+\alpha \beta_{\mathrm{ij}}+\varepsilon_{\mathrm{ijk}}$, where $\mu$ is the population mean, $\alpha_{i}$ is the fixed tag effect, $\beta_{j}$ is the random effect of tank, $\alpha \beta_{i j}$ is the interaction between both factors, and $\varepsilon_{\mathrm{ijk}}$ is the residual error. As the animals were sexed at the end of the experiment, a further GLM analysis for all growth and morphological traits was done using tag type and sex as fixed factors and tank as a random factor. To check the effect of tag position, the data were reanalyzed but using only tagged fish and using tag type $(\alpha)$, sex $(\delta)$ and tag position $(\gamma)$ as fixed factors and the tank $(\beta)$ as a random factor nested within tag type $\left(\mathrm{Y}_{\mathrm{ijk} \mathrm{m}}\right.$ $\left.=\mu+\alpha_{\mathrm{i}}+\beta_{\mathrm{ij}}+\delta_{\mathrm{k}}+\gamma_{1}+\alpha \delta_{\mathrm{ik}}+\alpha \gamma_{\mathrm{il}}+\delta \gamma_{\mathrm{kl}}+\alpha \delta \gamma_{\mathrm{ikl}}+\varepsilon_{\mathrm{ijklm}}\right)$. Finally, as weight and length were determined at different time points, a mixed ANOVA model with repeated measures using tag type, sex and tag position as fixed factors and the tank as a nested factor within the tag type was carried out. The Greenhouse-Geisse correction was used to evaluate time interaction effects. For the industrial trial, statistical differences between tagged and untagged fish were assessed by tstudent tests at each sampling point.

\subsection{Gene expression analysis}

Homogenization of samples, RNA isolation and cDNA synthesis pro cedures were carried out as previously described (Armesto et al., 2014; Armesto et al., 2015). For RNA isolation, a piece of $\sim 40 \mathrm{mg}$ wet weight of liver or brain was used. Real-time analysis was carried out on a $\mathrm{CFX}^{\mathrm{TM}}{ }^{\mathrm{TM}}$ Real-Time System (Bio-Rad). The qPCR reactions were accomplished in a $10 \mu \mathrm{l}$ 
volume containing cDNA generated from $10 \mathrm{ng}$ of original RNA template, $300 \mathrm{nM}$ each of specific forward and reverse primers, and $5 \mu \mathrm{l}$ of SYBR Premix Ex Taq (Takara, Clontech). Specific primers for heat shock protein 90 paralogs (hsp90aa and hpsp90ab), glucocorticoid receptors ( $g r l$ and $g r 2$ ), corticotrophin-releasing hormone ( $r f)$, CRF binding protein ( $c r f b p)$ and proopiomelanocortins (pomca and pomcb) were those reported previously (Benitez-Dorta et al., 2017; Benitez-Dorta et al., 2013; Manchado et al., 2008; Montero et al., 2015; Salas-Leiton et al., 2012). The amplification protocol used was as follows: initial 7 min denaturation and enzyme activation at $95^{\circ} \mathrm{C}, 40$ cycles of $30 \mathrm{~s}$ at $95^{\circ} \mathrm{C}, 15 \mathrm{~s}$ at $68^{\circ} \mathrm{C}$ and $30 \mathrm{~s}$ at $72^{\circ} \mathrm{C}$. Each PCR assay was performed in duplicate. Data were normalized using ubiquitin (ub52), which have been previously demonstrate to be suitable as reference genes in sole (Benitez-Dorta et al., 2013; Manchado et al., 2008; Salas-Leiton et al, 2012). Relative mRNA expression was determined using the $2^{-(\Delta \mathrm{Ct})}$ method (Livak and Schmittgen, 2001) using as calibrator the untagged control group at each sampling point. To assess significant differences, data were log-transformed and one-way ANOVA was carried out.

\section{Results and discussion}

\section{Effects of three tag types on loss rates and mortality in different weight classes of sole}

Previous tagging studies in flatfish (Pleuronectidae and Scophthalmidae) used the eyed-side as the best access route for tag injection since secondary infections and friction of wound against tank bottom were thus minimized (Moser et al., 2005; Oesau et al., 2013; Sparrevohn et al., 2014). However, this methodological approach is not feasible in sole due to the small size and large ellipticity of internal cavity. Hence, an alternative tagging procedure was tested and three different tags were evaluated in three different weight sizes. It is important to state that before 
starting experiments, all surgeons were trained for the use of the needle and inject the tag from the blind side through a small incision in the posterior part of the abdominal cavity (enough to inject each tag and to avoid a too large wound that would result in increased tag losses). Moreover, after tag injection, all animals were dispatched in self-cleaning tanks with a smooth bottom surface to maintain the microbiological quality of water combined with weekly prophylactic treatments and a reduced fish husbandry until wound healing for a fast recovery of animals

In the experiment 1 , the small weight class (average $0.33 \pm 0.07 \mathrm{~g}$; Table 1 ) was only tagged using the Nonatec tags due to the limited size of the abdominal cavity. The tag to fish weight ratio was $2.31 \pm 0.56 \%$ and tagging procedure resulted in $8 \%$ of tag losses similar to those data described in small seabass specimens $(2.2 \%$ tag/weight ratio) (Ferrari et al., 2014). In the middle weight class (average weight $0.82 \pm 0.27 \mathrm{~g}$ ), the tag to fish weight ratios ranged between 0.91 and 4.03 . Overall, tag loss rates, as determined in triplicate tanks, were significantly higher in mini$(15.1 \%)$ than nano-tagged $(5.2 \%)$ soles $\left(\chi_{(2)}^{2}=5.88, P<0.05\right)$ and the mortality was significantly higher in mini- $(15.0 \%)$ than Nonatec-tagged fish $(3.3 \%)\left(\chi_{(2)}^{2}=6.50, P<0.05\right)$. In the large weight class $(2.00 \pm 0.92 \mathrm{~g}$; Table 1$)$, the tag to fish weight ratios ranged between 0.41 and 1.81 and both the tag losses and mortality were very low (2-4\% in both cases) without differences between tag types. Non-parametric correlation analysis showed a very weak and negative association between fish weight and tag loss $(-0.13 ; P<0.05)$. Some previous studies indicated that tag loss and mortality rates were inversely correlated with fish size due to tag to body weight ratio and the higher capacity of larger fish to cope with manipulation (Acolas et al., 2007; Larsen et al., 2013; Mahapatra et al., 2001; Navarro et al., 2006; Soula et al., 2011). The small differences identified in this study among tags indicate that our methodology was robust 
and only the tag/cavity length appeared as the main factor influencing survival rates. If we take into account the optimal retention rates observed for nano tags both in the middle and large class, their robustness for signal recording and detection as well as their lower price compared with Nonatec tags, they appear as the best option be used in the pre-ongrowing stages of soles when grading activities become more intense while Nonatec tags would be advisable only in those trials involving very small size fish.

As five persons/surgeons participated in the tagging sessions of experiment 1, a possible effect of surgeon skills to inject the tag was also tested. Each surgeon tagged between 14.0 and $28.4 \%$ of fish in each experimental group and no significant differences between surgeons were observed for tag loss or mortality rates for any tag type $(P>0.05)$. It should be noted that, in our case, all surgeons were previously trained before proceeding with the trials. This is particularly important when small fish are used and for which tag retention rates can be highly dependent on individual surgeons (Richard et al., 2013).

To test the suitability of this methodology at industrial scale (experiment 3), four sole batches of a genetic breeding program were tagged using the mini transponders. The average weight for batches ranged between 1.7 and $6.0 \mathrm{~g}(\mathrm{n}=428-786)$. As can be observed in Table 1, tag loss rates increased in smaller fish ranging between 3.3 and $9.1 \%$ with mortality rates were low (0-1.9\%). These results confirmed those at experimental scale demonstrating the suitability of this approach to be implemented in selective breeding programs in sole.

\section{Effects of three tag types on growth traits in middle weight class of sole}

To assess the effects of tags on growth, soles of a middle weight class were tagged using Nonatec, nano- and mini-transponders in experiment 2. As Nonatec tags were read by a different 
device from nano- and mini- tags, soles were separated in triplicate tanks by tag type and a similar number of untagged fish was added. Average weight across all tanks was $1.03 \pm 0.28 \mathrm{~g}$ and no significant differences in weight or length was detected at the beginning of the trial across tag types or with respect to untagged fish.

Cumulative mortality through the experiment 2 a was $6.7 \%$. At 20 days, only a larger size of mini transponder tagged soles compared with untagged fish was detected (Table 2). During the rest of the trial (at 36 and 57), no differences in weight (Table 2), length (Supp. File 3) or area (Fig. 1) between tag types or for untagged fish were recorded. Moreover, no differences in SGR between tagged and untagged fish or between tag types were found. However, a smaller SGR57 value was observed in the last sampling (due to a reduced weight gain between 36 and $57 \mathrm{~d}$ ) that was even more evident in Nonatec tagged soles probably associated to intense fish handling (Fig. 1). These data agree with other reports that did not observe any effect on growth or behavior when animals were correctly managed and the tag properly inserted with only impaired growth in small size classes and during the initial period until wound healing (Cousin et al., 2012; Navarro et al., 2006; Ombredane et al., 1998; Richard et al., 2013).

To carry out a more accurate statistical analysis about the effects of tags on growth, the sex factor was considered into the evaluation model using all tagged and untagged soles at the end of the experiment. Males percentages ranged between 59.5, 66.7, 67.6 and $60.5 \%$ in mini, nano, Nonatec and untagged groups, respectively. The ANOVA analysis showed that males were statistically smaller than females in weight $\left(\mathrm{F}_{(1,466)}=16.80, P=0.002\right)$, length $\left(\mathrm{F}_{(1,465)}=17.94\right.$, $P=0.002)$ and width $\left(\mathrm{F}_{(1,466)}=9.68, P=0.012\right)$ (Fig. 2). This is the first time that these sexrelated differences are reported in such young sole stages (from 120 days post-hatch) in a similar way to small specimens of seabass (Ferrari et al., 2014) and zebrafish (Cousin et al., 2012). 
These sex-related differences have a clear impact on hatchery protocols since early size grading actions will bias sex ratios towards females and can change population dynamics. Moreover, significant differences among tag types for weight $\left(\mathrm{F}_{(3,466)}=6.39, p=0.026\right)$ were found with nano-tagged soles being bigger than untagged and Nonatec-tagged soles (Fig. 2). We hypothesize that added tag weight could benefit soles for movements on the bottom increasing the stability against the water flow in the self-cleaning tanks as it was also proposed for plaice (Arnold and Holford, 1978).

To check if tag position within the abdominal cavity might also modify growth performance, the position of each tag was established by using X-rays pictures (Supp. file 1 and 2). Statistical analysis indicated that tags did not distribute randomly in the abdominal cavity $\left(\chi^{2}=29.50, P\right.$ $<0.05)$. Mini- and Nano tags were mainly located in the posterior part of the abdominal cavity (70.0 and 60.3\%, respectively) immobilized in their positions and hardly in the medium part (4.8 and $10.6 \%$, respectively) (Fig. 3). In contrast, Nonatec tags were equally located in the three positions within the abdominal cavity (ranging between 27 and 39.2\% in anterior and posterior sections, respectively). Dissection of the fish confirmed positions and showed that the tags located in the anterior part were just below the septum that separates the organs between both sides (ocular and blind) of sole. To test if tag position had any effect on weight gain, a mixed ANOVA with repeated measures using tagged fish only was carried out. A significant main effect of $\operatorname{sex}\left(F_{(1,208)}=7.95, P=0.005\right)$ and tag type $\left(F_{(2,208)}=5.84, P=0.003\right)$ was found as previously observed but not for tag position $F_{(2,208)}=2.88, P=0.058$ (Fig. 4). However, a significant interaction between time*tag type $\left(\mathrm{F}_{(3.35,348.8)}=2.56, P=0.048\right)$ and time*tag position $\left(\mathrm{F}_{(3.35,348.8)}=3.26, P=0.018\right)$ was found with a reduced weight gain in soles with Nonatec tags and those tags located in posterior section of abdomen. In the case of length, significant 
differences were only observed between sexes $\left(\mathrm{F}_{(1,208)}=7.89, \mathrm{p}=0.005\right)$. Our main hypothesis is that these tags applied in the small weight class fish could slightly displace the balance point requiring a small acclimation period when compared with other anterior or medium position. Moreover, the delayed growth of Nonatec-tagged fish in comparison with the other two tag types could be mostly explained by the intense fish manipulation of these small specimens to ensure that tags were correctly read although a possible effect of the tag displacement inside the cavity and consequently a continuous adaptation to balance the position could not be discarded.

When tagging effects was tested at industrial scale using mini transponders (Experiment 3), no significant differences in weight between tagged fish and untagged fish were observed at the beginning of the trial or at 39 and 81 days after starting the experiment (Fig. 6). At 113 days, tagged fish were significantly heavier than untagged ones $(8.9 \pm 4.9$ vs $6.9 \pm 3.2 \mathrm{~g}$, respectively). At 148 days, the tagged group tended to be heavier although the differences were not significant due to a high dispersion typical of ungraded populations in sole (11.0 \pm 6.4 vs $12.5 \pm 7.6 \mathrm{~g})$. All these data confirm the experimental observations and highlight the suitability of the tagging procedure to evaluate growth under RAS production model in sole.

\section{Effects of three tag types on morphological traits}

To assess if PIT tags had any effect on the typical ellipsoid morphology of sole, the area of the ocular side at the end of the experiment in tagged soles was also tested.

A mixed ANOVA detected significant effects of tag type $\left(\mathrm{F}_{(2,221)}=5.55, P=0.008\right)$, sex $\left(\mathrm{F}_{(1}\right.$, 221) $=6.33, P=0.013)$ and tag location $\left(\mathrm{F}_{(2,221)}=3.99, P=0.020\right)$ with a smaller surface for those soles with tags in posterior position (Fig. 5). The nano-tagged fish appeared slightly larger than the other three groups and females larger than males. Moreover, other variables such as 
circularity, aspect ratio (AR) and ellipticity were determined at the final of biometry in Experiment 2a (at 57 days, Table 3). No significant differences in circularity and ellipticity were found between tag types and with respect to the untagged fish. Intriguingly, the AR revealed significant differences between tag types $\left(\mathrm{F}_{(3,467)}=8.02, P=0.016\right)$. Untagged fish showed a higher AR value than in mini and nano-tagged fish due to significant differences in the minor axis. These differences were not observed when the total length/width ratio was calculated. No effect of tag position was observed on morphological traits.

\section{Effects of three tag types on the expression of stress-related genes}

To evaluate the effect of the three types of tags on stress responses, the expression levels of six genes related to the HPI axis (pomca, pomcb, crf, crbp, grl and gr2) and two genes related with cellular stress defences (hsp90aa and hsp90ab) were quantified. At 2 days after tagging (dat), the pomca mRNA levels were higher in the brain of all tagged fish than in control groups. In contrast, reduced crf transcript abundance was found in Nonatec- and nano-tagged fish in comparison with the control soles (Fig. 7). At 11 dat, no significant difference between groups were found. This regulation is indicative of an acute stress response, in which the release of the adrenocorticotropic hormone activates corticosteroid biosynthesis, mainly cortisol, that in turn, would exert a negative feedback on $\mathrm{crf}$ biosynthesis to restore the system homeostasis. Although previous studies indicated the crfbp as a key gene exerting negative feedback on CRF actions (Salas-Leiton et al., 2012; Wunderink et al., 2011), the lack of response found in this study suggests the transcriptional regulation of $\mathrm{CRF}$ as the main mechanism to regulate the homeostatic response to this stress.

In the liver, hsp90aa mRNA levels were higher in tagged fish than in the control group at 2 dat although the difference was only significant for mini-tagged fish probably the damages induced 
by the larger size of this tag occupying most of the abdominal cavity. At 11 dat, higher transcript levels of $h s p 90 a b$ and $g r 2$ was found in nano-tagged sole when compared with untagged fish. Also, reduced grl mRNA levels in mini-tagged soles were detected when compared with the untagged fish (Fig. 8). These higher mRNA levels of $h s p 90 a b$ and $g r 2$ in the nano-tagged group could reflect metabolic changes in fish with the fastest growth rates whereas the reduction of $g r l$ mRNAs might be a recovery response after the acute stress as previously reported (BenitezDorta et al., 2017).

\section{Conclusion}

This study demonstrates that the three tag types can be successfully used in different weight classes of sole with optimal retention rates without any negative effects on growth and morphology. The tagging procedure was also carried out at industrial scale demonstrating its suitability for large genetic breeding schemes. The longitudinal analysis revealed the sex and tag position as important traits modulating growth performance. Gene expression indicated an acute stress response with the activation of HPI axis and cellular stress defenses just after tagging but animals had fully recovered at 11 days after tag injection.

\section{Acknowledgements}

The authors are grateful to Manuel Aparicio, Ana Manuela Crespo, Nuria Martin and staff from CUPIMAR for their valuable help in fish samplings and sample preparations. This research has been funded by INIA and EU through the FEDER 2014-2020 "Programa Operativo de Crecimiento Inteligente" project RTA2013-00023-C02-01. 
Figure 1. Left: Specific growth rate (SGR) for each tag type (Mini, nano and nonatec) and untagged fish at 20, 26 and 57 days in the medium weight class. Mean $(n=3)+S D$ is indicated. Right: Area of the eyed-side for each tag type and untagged fish at 57 days.

Figure 2. Top: Weight, length and width in males and females for tagged and untagged at 57 days. The asterisk denotes significant differences between sexes. Sex-corrected weight for each tag type and untagged fish at 57 days. The letters denote significant differences between the groups.

Figure 3. Distribution of each tag (Mini, Nano and Nonatec) in the anterior, medium or posterior part of abdominal cavity.

Figure 4. Estimated marginal means as determined ANOVA for repeated measures using tagged specimens for tag type (Mini, Nano and Nonatec), tag location (anterior, medium or posterior) and sex (male, female).

Figure 5. The Area of the ocular side at 57 days. Mean + SD for tag type (Mini, nano and nonatec), tag location (anterior, medium or posterior) and sex (male, female) is shown. The letters or asterisk denote significant differences between the groups.

Figure 6. Weight of mini-tagged and untagged soles at industrial scale. Mean $(n=50)+$ SD at 39, 81, 113 and 148 days is shown. The asterisk denotes significant differences between both groups at such sampling point. 
Figure 7. Relative expression levels of pomca, pomcb, $c r f$ and $c r f b p$ in the brain of soles tagged with Mini, nano and Nonatec and untagged fish at 2 (top) and 11 (bottom) days post tagging (dpt). Data were expressed as the mean fold change (mean $+\mathrm{SEM}, \mathrm{n}=3$ ) from the calibrator (untagged fish). The letters or asterisk denote significant differences between the groups.

Figure 8. Relative expression levels of $h s p 90 a b, h s p 90 a a, g r 1$, and $g r 2$ in the brain of soles tagged with mini, nano and Nonatec and untagged fish at 2 (top) and 11 (bottom) days post tagging (dpt). Data were expressed as the mean fold change (mean + SEM, $n=3$ ) from the calibrator (untagged fish). The letters or asterisk denote significant differences between the groups.

Supp. File 1. Tagging procedure. A) Comparison of tags used in the study. From top to bottom, mini, nano and Nonatec transponders; B) comparison of middle weight class, Nano tag and needle use for incision; C) Selected area to measure eyed-side surface; D) Injection of Nano tag; E) Introduction of Mano-tag in the abdominal cavity by using forceps; F) Rx of tagged and untagged fish.

Supp. File 2. Tag position in abdominal cavity. Mini, Nano, and Nonatec tags in anterior, medium and posterior position are shown. Rx image and position after dissection is shown.

Supp. File 3. Length of Mini-, Nano-, and Nonatec-tagged soles and untagged specimens at 0 , 20, 36 and 57 days. Mean $(\mathrm{cm}) \pm \mathrm{SD}$ and number of individuals in each group are shown. 


\section{References}

Acolas, M., Roussel, J., Lebel, J., Baglinière, J., 2007. Laboratory experiment on survival, growth and tag retention following PIT injection into the body cavity of juvenile brown trout (Salmo trutta). Fish Res. 86, 280-284.

Anguís, V., Cañavate, J.P., 2005. Spawning of captive Senegal sole (Solea senegalensis) under a naturally fluctuating temperature regime. Aquaculture. 243, 133-145.

Armesto, P., Campinho, M.A., Rodriguez-Rua, A., Cousin, X., Power, D.M., Manchado, M., Infante, C., 2014. Molecular characterization and transcriptional regulation of the $\mathrm{Na}$ $+/ \mathrm{K}+$ ATPase alpha subunit isoforms during development and salinity challenge in a teleost fish, the Senegalese sole (Solea senegalensis). Comp. Biochem Physiol B Biochem Mol Biol. 175, 23-38.

Armesto, P., Infante, C., Cousin, X., Ponce, M., Manchado, M., 2015. Molecular and functional characterization of seven $\mathrm{Na} / \mathrm{K}$ ATPase beta subunit paralogs in Senegalese sole (Solea senegalensis Kaup, 1858). Comp. Biochem Physiol B Biochem Mol Biol. 182, 14-26.

Arnold, G.P., Holford, B.H., 1978. The physical effects of an acoustic tag on the swimming performance of plaice and cod. J. Cons. Explor. Mer. 38, 189-200.

Baras, E., Malbrouck, C., Houbart, M., Kestemont, P., Mélard, C., 2000. The effect of PIT tags on growth and physiology of age-0 cultured Eurasian perch Perca fluviatilis of variable size. Aquaculture. 185, 159-173.

Bégout, M.L., Bau, F., Acou, A., Acolas, M.L., 2016. Methodologies for investigating diadromous fish movements: conventional, PIT, acoustic and radio tagging and tracking. in: Morais, P., Daverat, F. (Eds.), An Introduction to Fish Migration. CRC Press, Boca Raton, FL, USA pp. 214-250.

Benitez-Dorta, V., Caballero, M.J., Izquierdo, M., Manchado, M., Infante, C., Zamorano, M.J., Montero, D., 2013. Total substitution of fish oil by vegetable oils in Senegalese sole (Solea senegalensis) diets: effects on fish performance, biochemical composition, and expression of some glucocorticoid receptor-related genes. Fish Physiol Biochem. 39, 335-349.

Benitez-Dorta, V., Caballero, M.J., Betancor, M.B., Manchado, M., Tort, L., Torrecillas, S., Zamorano, M.J., Izquierdo, M., Montero, D., 2017. Effects of thermal stress on the expression of glucocorticoid receptor complex linked genes in Senegalese sole (Solea senegalensis): Acute and adaptive stress responses. Gen Comp Endocrinol. 252, 173-185.

Cousin, X., Daouk, T., Pean, S., Lyphout, L., Schwartz, M.E., Begout, M.L., 2012. Electronic individual identification of zebrafish using radio frequency identification (RFID) microtags. J Exp Biol. 215, 2729-2734.

Ferrari, S., Chatain, B., Cousin, X., Leguay, D., Vergnet, A., Vidal, M.-O., Vandeputte, M., Begout, M.L., 2014. Early individual electronic identification of sea bass using RFID microtags: A first example of early phenotyping of sex-related growth. Aquaculture. 426-427, 165-171.

Ibarra-Zatarain, Z., Fatsini, E., Rey, S., Chereguini, O., Martin, I., Rasines, I., Alcaraz, C., Duncan, N., 2016. Characterization of stress coping style in Senegalese sole (Solea senegalensis) juveniles and breeders for aquaculture. R Soc Open Sci. 3, 160495.

Jepsen, N., Schreck, C., S., C., Thorstad, E.B., 2005. A brief discussion on the $2 \%$ tag/bodymass rule of thumb. in: Spedicato, M.T., Lembo, G., Marmulla, G. (Eds.), Aquatic telemetry: advances and applications. FAO/COISPA, Ustica, Italy, pp. 255-259. 
Larsen, M.H., Thorn, A.N., Skov, C., Aarestrup, K., 2013. Effects of passive integrated transponder tags on survival and growth of juvenile Atlantic salmon Salmo salar. Animal Biotelemetry. 1, 19.

Livak, K.J., Schmittgen, T.D., 2001. Analysis of relative gene expression data using real-time quantitative PCR and the 2(-Delta Delta C(T)) Method. Methods. 25, 402-408.

Mahapatra, K., Gjerde, B., Reddy, P., Sahoo, M., Jana, R., Saha, J., Rye, M., 2001. Tagging: on the use of passive integrated transponder PIT tags for the identification of fish. Aquacult Res. 32, 47-50.

Manchado, M., Infante, C., Asensio, E., Planas, J.V., Cañavate, J.P., 2008. Thyroid hormones down-regulate thyrotropin beta subunit and thyroglobulin during metamorphosis in the flatfish Senegalese sole (Solea senegalensis Kaup). Gen Comp Endocrinol. 155, 447-455.

Marquez, L., Morales, G., Sáenz de Rodrigáñez, M., Almansa, E., Moyano, F., Díaz, M., 2015. Comments on the calculation of the specific growth rate in experiments with untagged individuals. Sci Mar. 79, 505-508.

Montero, D., Benitez-Dorta, V., Caballero, M.J., Ponce, M., Torrecillas, S., Izquierdo, M., Zamorano, M.J., Manchado, M., 2015. Dietary vegetable oils: effects on the expression of immune-related genes in Senegalese sole (Solea senegalensis) intestine. Fish Shellfish Immunol. 44, 100-108.

Morais, S., Aragão, C., Cabrita, E., Conceição, L.E.C., Constenla, M., Costas, B., Dias, J., Duncan, N., Engrola, S., Estevez, A., Gisbert, E., Mañanos, E., Valente, L.M.P., Yufera, M., Dinis, M.T., 2016. New developments and biological insights into the farming of Solea senegalensis reinforcing its aquaculture potential. Rev. Aquacult. 6, 1-37.

Moser, M.L., Myers, M.S., Burke, B.J., O’Neill, S.M., 2005. Effects of surgically-implanted transmitters on survival and feeding behavior of adult English sole. in: Spedicato, M.T., Lembo, G., Marmulla, G. (Eds.), Aquatic telemetry: advances and applications. FAO/COSIPA, Ustica, Italy, pp. 269-274.

Navarro, A., Oliva, V., Zamorano, M.J., Ginés, R., Izquierdo, M.S., Astorga, N., Afonso, J.M., 2006. Evaluation of PIT system as method to tag fingerlings of gilthead seabream (Sparus auratus L.): effects on growth, mortality and tag loss. Aquaculture. 257, 309315.

Oesau, S., Thaller, G., Schulz, C., Tetens, J., 2013. Application of PIT tags for individual identification of turbot (Scophthalmus maximus). Archiv Tierzucht 56, 285-292.

Ombredane, D., Baglinière, J., Marchand, F., 1998. The effects of passive integrated transponder tags on survival and growth of juvenile brown trout (Salmo trutta L.) and their use for studying movement in a small rive. Hydrobiologia. 371/372, 99-106.

Richard, A., O'Rourke, J., Caudron, A., Cattanéo, F., 2013. Effects of passive integrated transponder tagging methods on survival, tag retention and growth of age-0 brown trout. Fish. Res. 145, 37-42.

Salas-Leiton, E., Anguis, V., Martin-Antonio, B., Crespo, D., Planas, J.V., Infante, C., Cañavate, J.P., Manchado, M., 2010a. Effects of stocking density and feed ration on growth and gene expression in the Senegalese sole (Solea senegalensis): potential effects on the immune response. Fish Shellfish Immunol. 28, 296-302.

Salas-Leiton, E., Anguis, V., Rodriguez-Rua, A., Cañavate, J.P., 2010b. Stocking homogeneous size groups does not improve growth performance of Senegalese sole (Solea senegalensis, Kaup 1858) juveniles: Individual growth related to fish size. Aquacult Eng. 43, 108-113. 
Salas-Leiton, E., Coste, O., Asensio, E., Infante, C., Cañavate, J.P., Manchado, M., 2012. Dexamethasone modulates expression of genes involved in the innate immune system, growth and stress and increases susceptibility to bacterial disease in Senegalese sole (Solea senegalensis Kaup, 1858). Fish Shellfish Immunol. 32, 769-778.

Soula, M., Navarro, A., Hildebrandt, S., Zamorano, M.J., Roo, J., Hernández-Cruz, C., Afonso, J.M., 2011. Evaluation of VIE (Visible Implant Elastomer) and PIT (Passive Integrated Transponder) physical tagging systems for the identification of red porgy fingerlings (Pagrus pagrus). Aquacult. Int. 20, 571-583.

Sparrevohn, C.R., Aarestrup, K., Stenberg, C., Righton, D., 2014. Scanning for PIT-tagged at sh in a coastal area using a sledge equipped with an RFID antenna. J. Fish Biol. 85, 523529.

Wunderink, Y.S., Engels, S., Halm, S., Yufera, M., Martinez-Rodriguez, G., Flik, G., Klaren, P.H., Mancera, J.M., 2011. Chronic and acute stress responses in Senegalese sole (Solea senegalensis): the involvement of cortisol, CRH and CRH-BP. Gen Comp Endocrinol. 171, 203-210. 
Table 1: Tag loss and mortality rates for three weight classes (Small, middle and big) at experimental and industrial scales. The initial mean weight $(\mathrm{g})$ and length $(\mathrm{cm}) \pm \mathrm{SD}$ for each weight class, tag type (Nonatec, nano and mini), the tag to fish weight ratio and the number of individuals (n) are indicated. Letter denote significantly difference between tag type in the middle weight class.

\begin{tabular}{|c|c|c|c|c|c|c|c|}
\hline $\begin{array}{l}\text { Size } \\
\text { class }\end{array}$ & Tag & Weight & Length & $\mathrm{n}$ & $\begin{array}{c}\text { Tag/fish } \\
\text { weight }(\%)\end{array}$ & $\begin{array}{c}\text { Tag loss } \\
(\%)\end{array}$ & Mortality(\%) \\
\hline Small & Nonatec & $0.33 \pm 0.07$ & $3.0 \pm 0.2$ & 25 & $2.31 \pm 0.56$ & 8.0 & 4.0 \\
\hline \multirow{3}{*}{$\begin{array}{l}\frac{0}{7} \\
\dot{z} \\
\sum\end{array}$} & Nonatec & $0.86 \pm 0.28$ & $3.9 \pm 0.5$ & 150 & $0.91 \pm 0.25$ & $10.0 \pm 3.5^{\mathrm{ab}}$ & $3.3 \pm 1.2^{\mathrm{a}}$ \\
\hline & Nano & $0.81 \pm 0.27$ & $3.8 \pm 0.5$ & 157 & $3.28 \pm 1.02$ & $5.2 \pm 3.0^{\mathrm{a}}$ & $6.4 \pm 4.2^{\mathrm{ab}}$ \\
\hline & Mini & $0.80 \pm 0.24$ & $3.8 \pm 0.4$ & 145 & $4.03 \pm 1.03$ & $15.1 \pm 4.6^{b}$ & $15.0 \pm 1.5^{b}$ \\
\hline \multirow{3}{*}{ 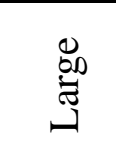 } & Nonatec & $2.07 \pm 0.96$ & $5.1 \pm 0.8$ & 50 & $0.41 \pm 0.15$ & 2.0 & 2.0 \\
\hline & Nano & $2.03 \pm 1.10$ & $5.0 \pm 0.9$ & 50 & $1.50 \pm 0.58$ & 2.0 & 2.0 \\
\hline & Mini & $1.87 \pm 0.66$ & $4.9 \pm 0.6$ & 50 & $1.81 \pm 0.62$ & 4.0 & 2.0 \\
\hline \multirow{4}{*}{ 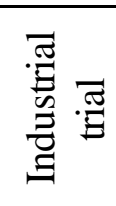 } & Mini & $6.0 \pm 4.4$ & $7.3 \pm 1.6$ & 428 & $0.80 \pm 0.56$ & 3.3 & 0.0 \\
\hline & Mini & $3.6 \pm 1.8$ & $6.2 \pm 1.0$ & 786 & $1.05 \pm 0.59$ & 7.3 & 0.6 \\
\hline & Mini & $1.8 \pm 1.1$ & $4.9 \pm 1.0$ & 683 & $2.19 \pm 1.08$ & 9.1 & 1.9 \\
\hline & Mini & $1.7 \pm 0.9$ & $4.8 \pm 0.8$ & 748 & $2.29 \pm 2.29$ & 9.0 & 1.5 \\
\hline
\end{tabular}


Table 2: Weight of mini-, nano-, and Nonatec-tagged soles and untagged specimens at 0, 20, 36 and 57 days. Mean $(\mathrm{g}) \pm \mathrm{SD}$ and number of individuals in each group are shown. Asterisks denote statistically significant differences between tagged and untagged fish.

\begin{tabular}{|l|c|c|c|c|c|c|c|c|}
\hline & & $n$ & W0 & & W20 & W36 & W57 \\
\hline Mini & Tagged & 84 & $1.05 \pm 0.31$ & & $1.49 \pm 0.51 *$ & $2.04 \pm 0.81$ & $2.51 \pm 1.23$ \\
\hline & Untagged & 89 & $0.99 \pm 0.26$ & $1.38 \pm 0.43$ & $1.97 \pm 0.75$ & $2.43 \pm 1.13$ \\
\hline & & & & & & & & \\
\hline Nano & Tagged & 87 & $1.08 \pm 0.32$ & $1.58 \pm 0.53$ & $2.20 \pm 0.86$ & $2.89 \pm 1.45$ \\
\hline & Untagged & 87 & $1.03 \pm 0.27$ & $1.45 \pm 0.49$ & $1.95 \pm 0.71$ & $2.36 \pm 0.98$ \\
\hline & & & & & & & \\
\hline Nonatec & Tagged & 74 & $0.97 \pm 0.26$ & $1.37 \pm 0.40$ & $1.95 \pm 0.65$ & $2.20 \pm 0.89$ \\
\hline & Untagged & 83 & $1.04 \pm 0.24$ & & $1.38 \pm 0.40$ & $1.94 \pm 0.67$ & $2.23 \pm 0.97$ \\
\hline
\end{tabular}


Table 3: Shape parameters (circularity, aspect ratio and ellipticity) of mini-, nano-, and nonatectagged soles and untagged specimens at 0,20,36 and 57 days. Mean values \pm SD and number of individuals in each group are shown.

\begin{tabular}{|l|l|c|c|c|c|}
\hline & & $\mathrm{n}$ & Circularity & Aspect Ratio & Ellipticity \\
\hline Mini & Tagged & 84 & $0.333 \pm 0.057$ & $2.724 \pm 0.106^{*}$ & $0.477 \pm 0.03$ \\
\hline & Untagged & 89 & $0.327 \pm 0.056$ & $2.765 \pm 0.090$ & $0.483 \pm 0.06$ \\
\hline & & & & & \\
\hline Nano & Tagged & 87 & $0.327 \pm 0.068$ & $2.711 \pm 0.109^{*}$ & $0.475 \pm 0.04$ \\
\hline & Untagged & 87 & $0.339 \pm 0.070$ & $2.752 \pm 0.096$ & $0.479 \pm 0.02$ \\
\hline & & & & & \\
\hline Nonatec & Tagged & 74 & $0.307 \pm 0.063$ & $2.707 \pm 0.091$ & $0.476 \pm 0.04$ \\
\hline & Untagged & 82 & $0.308 \pm 0.064$ & $2.714 \pm 0.093$ & $0.458 \pm 0.17$ \\
\hline
\end{tabular}




\section{Highlights}

- A methodology for internal tagging of small soles was developed and validated at experimental and industrial scales

- Optimal tag retention and mortality rates were obtained and no negative effects on growth or morphology were detected.

- Longitudinal analysis using internal tags revealed a major effect of sex, tag type and tag position on growth.

- Expression analysis indicated no signs of chronic stress linked to internal tags. 

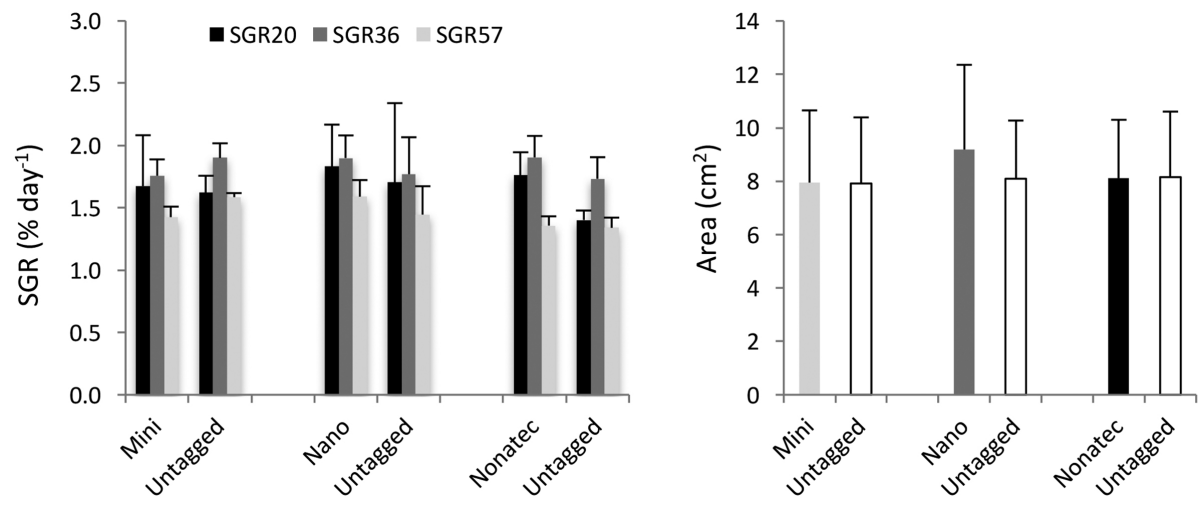

Figure 1 

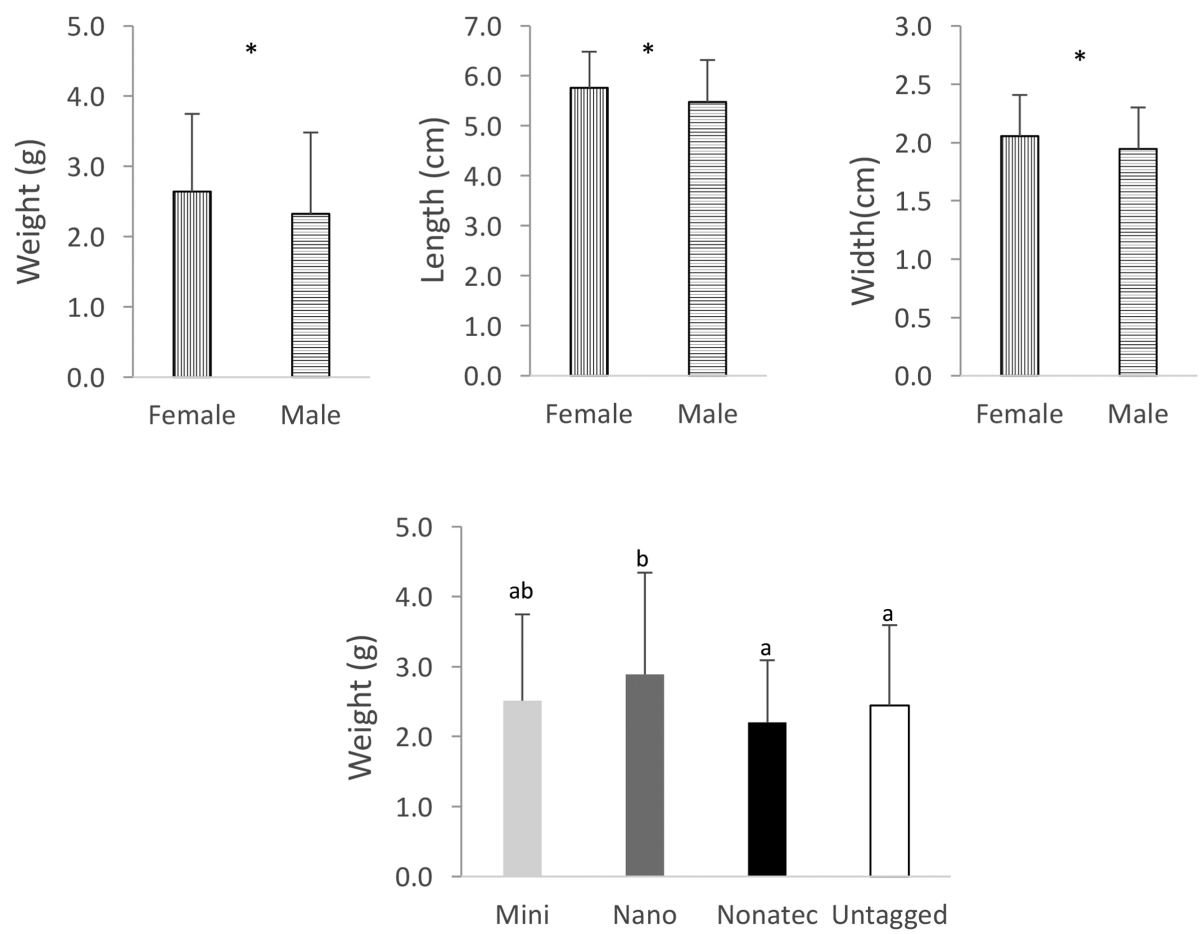

Figure 2 


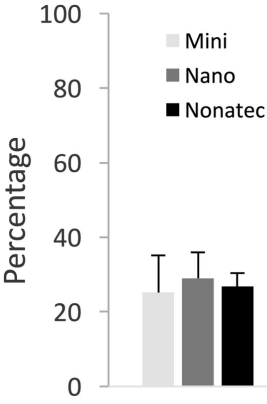

Anterior

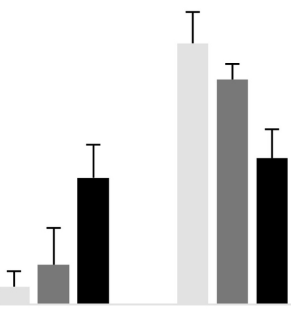

Medium

Figure 3 

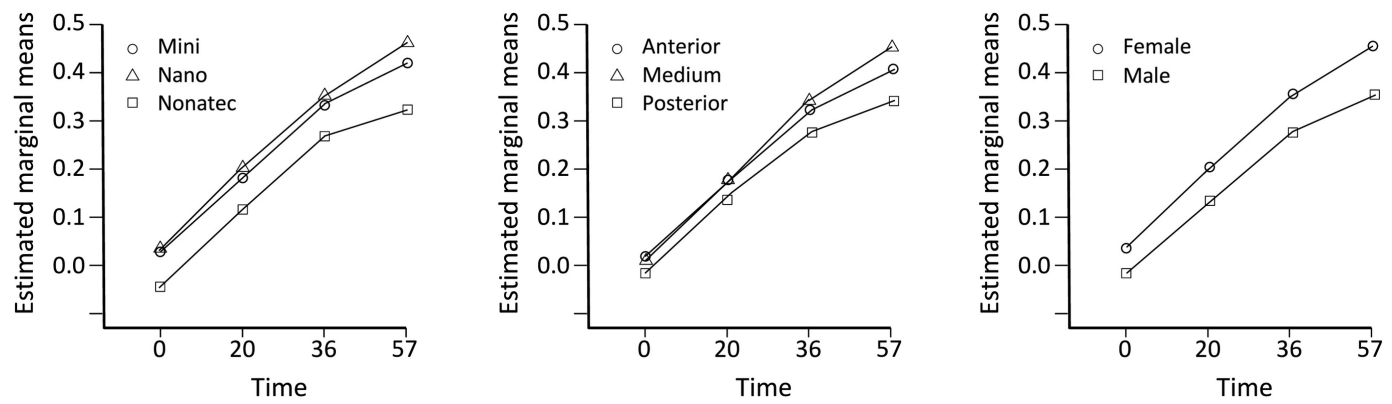

Figure 4 

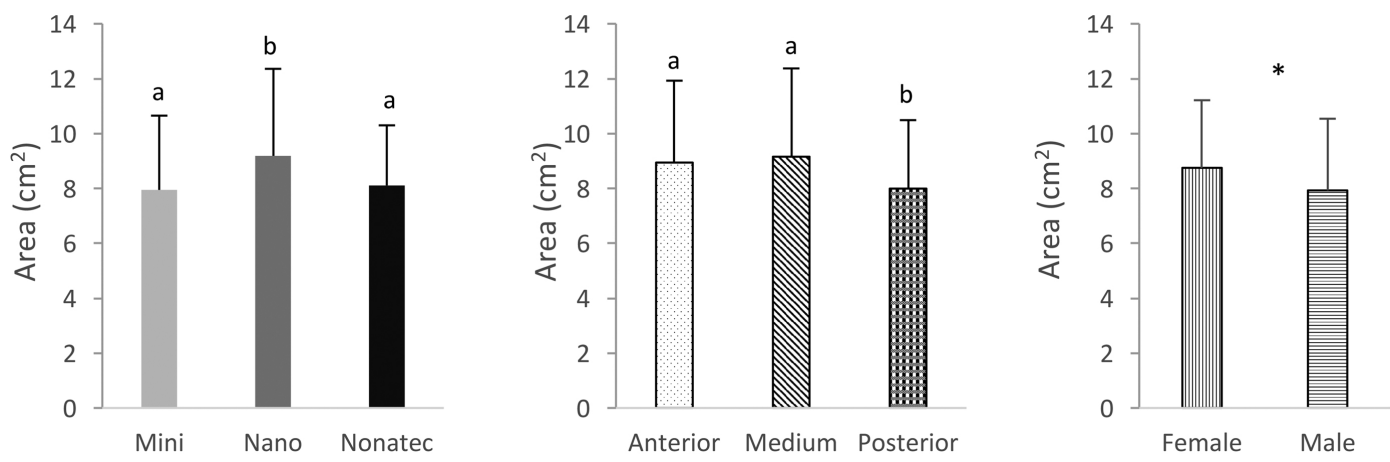

Figure 5 


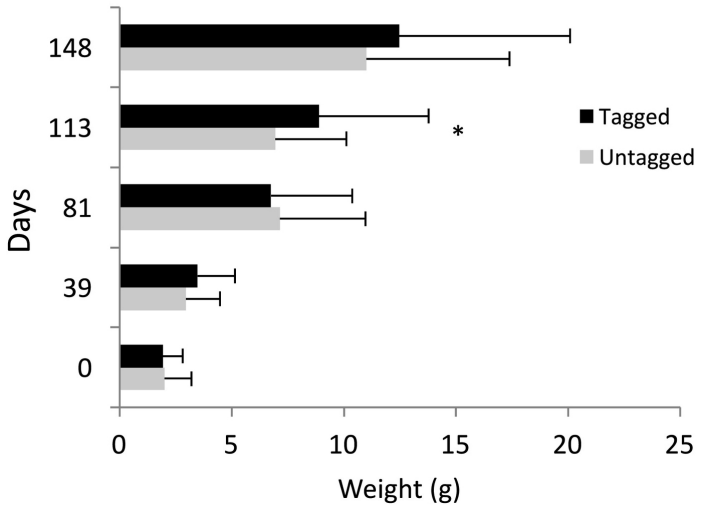

Figure 6 


\section{2 dat}
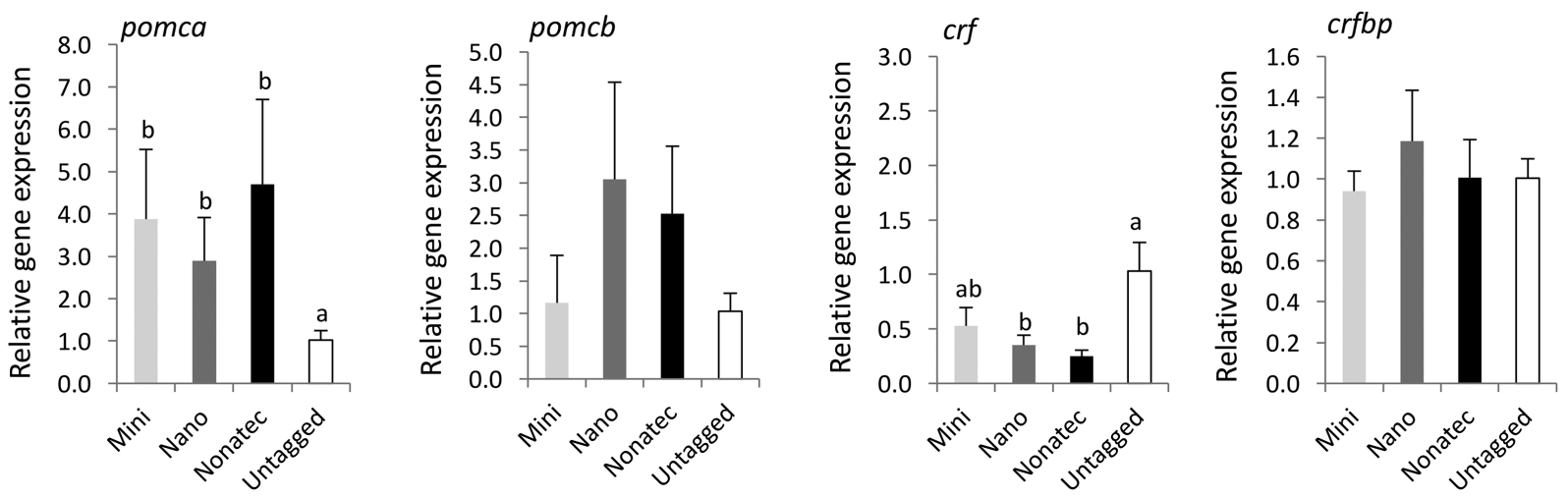

\section{1 dat}
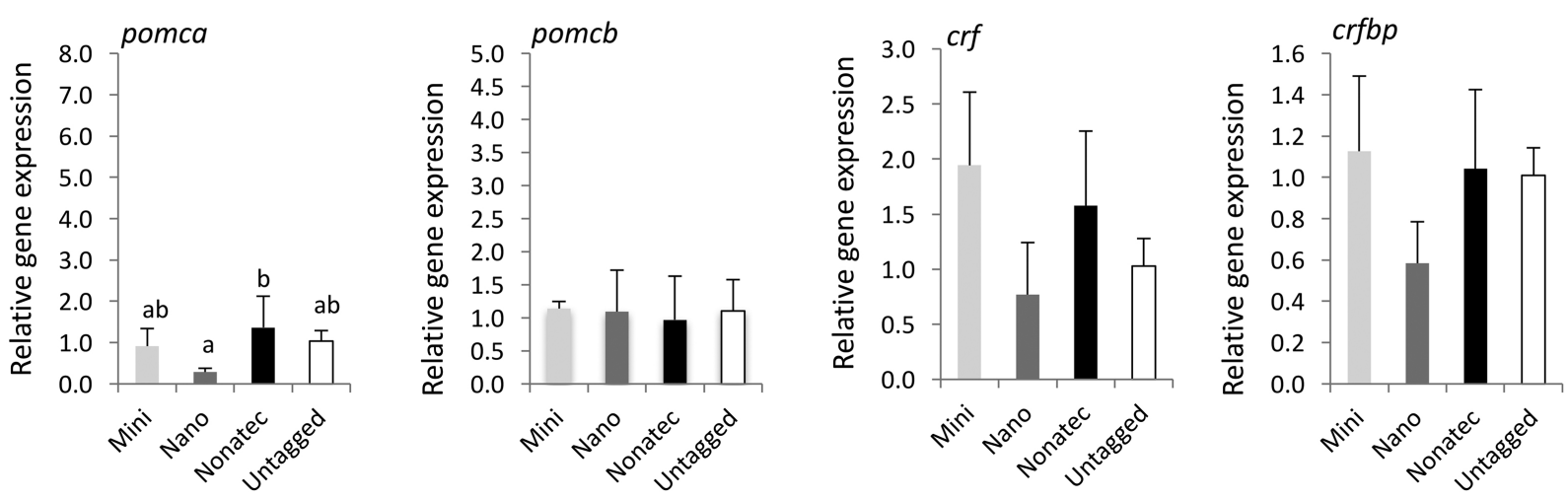

Figure 7 


\section{2 dat}
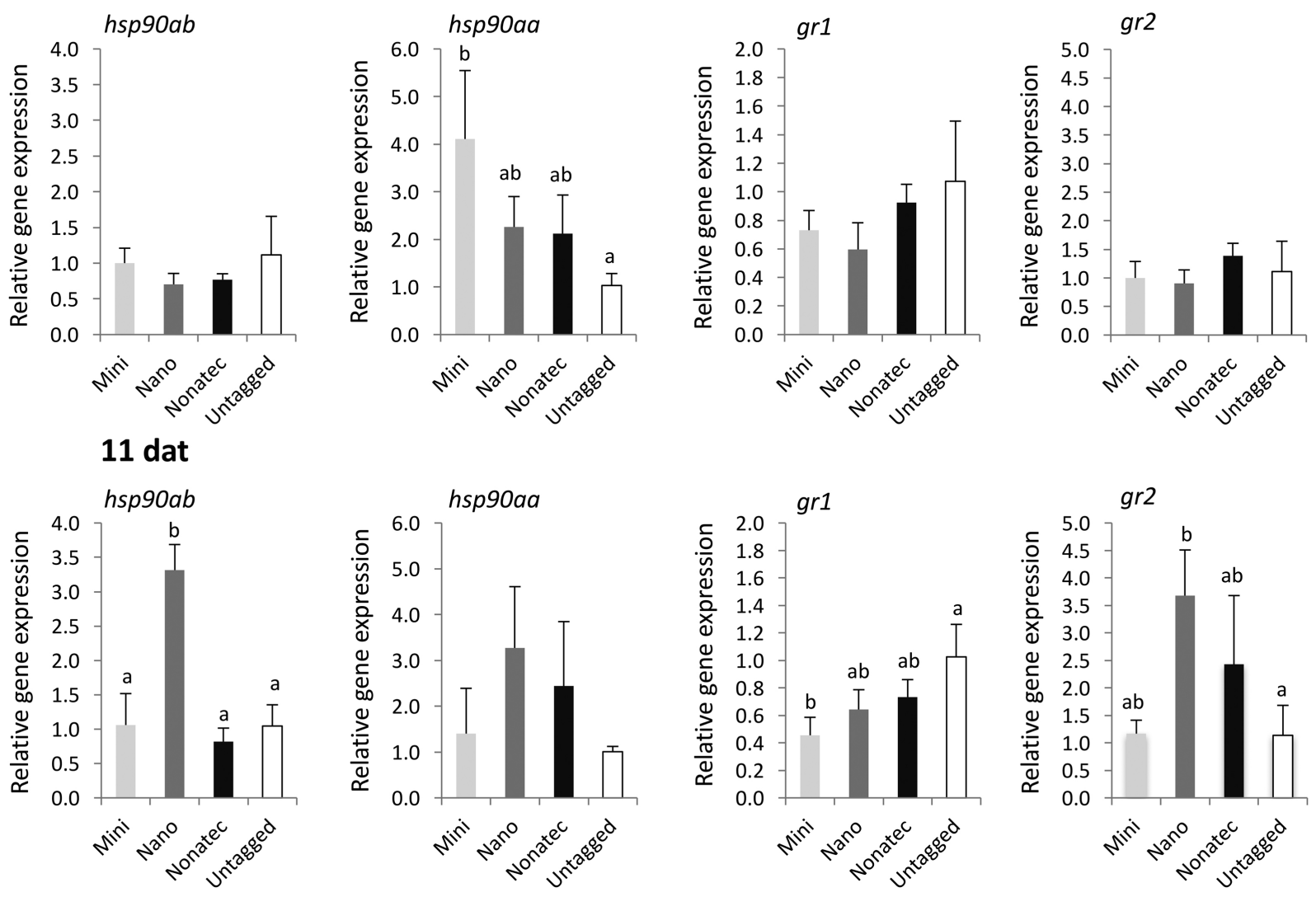

Figure 8 